\title{
Integral Terminal Sliding Mode Control for a Class of Nonaffine Nonlinear Systems with Uncertainty
}

\author{
Qiang Zhang, Hongliang Yu, and Xiaohong Wang \\ School of Electrical Engineering, University of Jinan, Jinan 250022, China \\ Correspondence should be addressed to Qiang Zhang; zhang_hongyu198023@163.com
}

Received 18 October 2013; Accepted 8 December 2013

Academic Editor: Xiaojie Su

Copyright (c) 2013 Qiang Zhang et al. This is an open access article distributed under the Creative Commons Attribution License, which permits unrestricted use, distribution, and reproduction in any medium, provided the original work is properly cited.

\begin{abstract}
This paper is concerned with an integral terminal sliding mode tracking control for a class of uncertain nonaffine nonlinear systems. Firstly, the nonaffine nonlinear systems is approximated to facilitate the desired control design via a novel dynamic modeling technique. Next, for the unmeasured disturbance of nonlinear systems, integral terminal sliding mode disturbance observer is presented. The developed disturbance observer can guarantee the disturbance approximation error to converge to zero in the finite time. Subsequently, based on approximated nonlinear model and the designed disturbance observer, the integral terminal sliding mode tracking control is presented for nonaffine nonlinear systems with uncertainty. Different from traditional terminal slidingmode control, this paper accomplishes finite convergence time for nonaffine nonlinear systems and avoids the singular problem in the controller design. Furthermore, the control system is forced to start on the terminal sliding hyperplane, so that the reaching time of the sliding modes is eliminated. Finally, two numerical simulation results are given to illustrate the effectiveness of the proposed method.
\end{abstract}

\section{Introduction}

In recent years, there has been significant progress in the area of designing controllers for nonlinear systems [1-10]. Most of the controllers developed in this context have the common assumption that the system to be controlled is affine; that is, the plant is linear according to the input variables, and the nonlinearities are linearly parameterized by unknown parameters. However, developing a systematic synthesis method for nonaffine nonlinear systems is still a challenging problem for now.

Sliding-mode control (SMC) is a well-known efficient control scheme which has been widely applied for both linear and nonlinear systems $[8,10-16]$. This control is also considered as an effective approach for control of the systems with uncertainties. However, the main disadvantage of SMC scheme is that the system states cannot reach the equilibrium point in finite time. So a new control scheme called terminal sliding-mode control (TSMC) is proposed to overcome this drawback utilizing nonlinear sliding surface. Nonlinear switching hyperplanes in TSMC can improve the transient performance substantially. Besides, compared with the conventional SMC with linear sliding manifold, TSMC offers some superior properties such as faster, finite time convergence and higher control precision. However, there exists an intrinsic singular problem in TSMC due to using fractional power functions as the sliding hyperplane [17-23]. Thus, overcoming the singular problem becomes a considerable topic for TSMC. Unfortunately, most of nonsingular TSMC methods $[22,23]$ are available only for affine nonlinear systems, especially robotic manipulators received the most attention. Nevertheless, these nonsingular TSMC schemes lack strict theoretical analysis and are only suitable for affine nonlinear systems. It is a worthwhile note that only a few researches discuss the control of nonaffine nonlinear systems even if allowing singularity in the TSMC. Therefore, nonsingular TSMC of nonaffine nonlinear systems needs to be investigated by a new technique.

The uncertainty is inherent in practical systems. Designing controller capability of handing uncertainty is of practical interest and is academically challenging. Neural networks (NNs) have been proposed recently as an adaptive controller for nonlinear systems. By the use of their universal approximation capability, the adaptive controller based on 
neural networks can be designed without significant prior knowledge of the system dynamics [24-28]. Although it is widely accepted that the parameterized neural network is capable of approximating linear or nonlinear mapping by adequately choosing network structures and training methods, a challenging problem for designers is to select an appropriate structure for balancing the number of rules and the approximation accuracy. If the network size is chosen too small, it is impossible to assure the approximation to converge to an acceptable level due to the limited nodes. On the other hand, if overdetermined nodes are given, the computational burden is huge and the waste of computation resource implies its impracticality for real-world applications.

Based on the above works, this paper is to develop an integral terminal sliding mode control approach for a class of nonaffine nonlinear systems with uncertainty, parameters perturbation, and external disturbances. The organization of this paper is as follows. Following the introduction, the problem formulation is described briefly, some assumptions which will play a basic role in our analysis are introduced in Section 2. To facilitate the desired control design, a novel dynamic modeling technique has been proposed for the nonaffine nonlinear systems in Section 3. The integral terminal sliding mode disturbance observer is presented in Section 4 . Section 5 proposes integral terminal sliding mode control based on disturbance observer, and then integral terminal sliding mode control is designed for uncertain nonlinear systems with control singularity. Simulation studies are shown in Section 6 to demonstrate the effectiveness of our proposed approaches. Finally, conclusions are drawn in Section 7.

\section{Problem Formulation}

Consider a class of uncertain nonaffine nonlinear systems that can be expressed in the following form:

$$
\begin{gathered}
\dot{x}(t)=f_{1}(x(t), u(t))+\Delta f_{1}(x(t), u(t))+d(t), \\
y(t)=x(t),
\end{gathered}
$$

where $x(t)=\left[x_{1}(t), \ldots, x_{n}(t)\right] \in \mathbb{R}^{n}$ is the state vector of the system in the normal form which is assumed available for measurement, $y(t) \in \mathbb{R}^{n}$ is the output vector, $u(t) \in \mathbb{R}^{n}$ is control input vector, and $f_{1}(x(t), u(t)) \in \mathbb{R}^{n}$ is known smooth vector fields. $\Delta f_{1}(x(t), u(t))$ is assumed to be continuous of $x(t)$ denoting the the system uncertainty, which contains structural and modeling error. $d(t) \in \mathbb{R}^{n}$ is external disturbance.

After combining the uncertainty and disturbance together, the nonlinear system (1) can be rewritten as

$$
\begin{gathered}
\dot{x}(t)=f_{1}(x(t), u(t))+D(x(t), u(t), t), \\
y(t)=x(t),
\end{gathered}
$$

where $D(x, u, t)=\Delta f_{1}(x(t), u(t))+d(t)$ is called compound disturbance.

To achieve the proposed control objective, the following assumptions are required.
Assumption 1. There exist known positive constants $\check{\epsilon}$ such that for all $t \in R^{+},\|D(x, u, t)\| \leqslant \check{\epsilon}$.

Assumption 2. Consider $\|\Delta u\| \in[0, \delta]$ and $0 \leqslant\|\partial f / \partial u\| \leqslant \check{\delta}$, where $\delta$ and $\check{\delta}$ are two finite positive constants.

Remark 3. In many actual process control systems and flight control systems, $\|\Delta u(t)\| \in[0, \delta]$ is a physical restriction of many practical systems because their states and outputs (actuators) cannot change too fast because of system "inertia." So, Assumption 2 is reasonable.

In this paper, the control objective is to design the disturbance-observer-based integral terminal sliding mode tracking control and make the system output follow a given desired output of the nonlinear system in the presence of uncertainty and external disturbance. For the desired tracking signal $y_{d}$, the proposed integral terminal sliding mode control must ensure that all closed-loop signals are convergent in the finite time.

\section{Novel Nonaffine Nonlinear Approximation}

The problem of controlling the plants characterized by models that are nonaffine in the control input vector is a difficult one. Especially for the tracking control, the liberalization may result in the design of sufficiently accurate controllers in the case of stabilization around the operating point, in the case of tracking of desired trajectories the problem becomes much more difficult, because the linearized model is timevarying. Hence, there is a clear need for the development of systematic control design techniques for nonlinear models that are nonaffine in $u$ and that are suitable for the case of tracking of desired trajectories.

For the nonaffine nonlinear model (2), the Taylor expansion of the nonlinear function $f_{1}(x(t), u(t))$ with respect to $u(t)$ around $u(t-\tau)$ can result in

$$
\dot{x}=f_{1}(x, u(t-\tau))+g(x, u(t-\tau)) \Delta u+O(\cdot)+D,
$$

where $\Delta u=u-u(t-\tau), g(x, u(t-\tau))=$ $\left.\left(\partial f_{1}(x, u) / \partial u\right)\right|_{u=u(t-\tau)}$, and the remainder $O(\cdot)=$ $[\Delta u]^{T} f_{d d} \Delta u / 2$ is bounded by

$$
\|O(\cdot)\| \leqslant \frac{r_{p}\|\Delta u\|^{2}}{2},
$$

where $f_{d d}=\left.\left(\partial^{2} f_{1}(x, u) / \partial^{2} u\right)\right|_{u=\zeta}$ and $\zeta$ is a point between $u$ and $u(t-\tau)$. Let $0 \leq\left\|f_{d d}\right\| \leq r_{p}$ with $r_{p}$ as a finite positive number. The parameter $\tau>0$ is the updating input. It may be chosen as the sampling-time in a sampled-data control system or as an integer multiple of the sampling-time. A better choice of the parameter $\tau$ is the sampling because a larger $\tau$ may lead to an inaccurate approximation when the system function $f_{1}(x, u)$ varies quickly.

Equation (3) can be representation as the following form:

$$
\dot{x}=f(x, u(t-\tau))+g(x, u(t-\tau)) u+O(\cdot)+D(x, u, t),
$$


where $f(x, u(t-\tau))=f_{1}(x, u(t-\tau))-f_{d}(x, u(t-\tau)) u(t-\tau)$. Convenient for the following statements, $u(t-\tau)$ is defined as $v(t)$; then (3) can be described as follows:

$$
\dot{x}=f(x, v)+g(x, v) u+O(\cdot)+D(x, u, t) .
$$

Remark 4. In Assumption 2, $\|\Delta u\|$ should not be too large in order to limit the approximation error of model (3) for a computed $u(t)$. Therefore, to approximation accuracy, Assumption 2 must be satisfied. The significance of the Assumption 2 has been explained in Remark 3.

Remark 5. The traditional model simplification method does not global. It can be seen that (6) is a time-varying simplified model. The method which is proposed in this subsection can achieve the global approximation for nonlinear systems (2). So the proposed simplified model method can effectively solve the tracking control problem using affine nonlinear control strategy, such as sliding mode control, outputfeedback control, and backstepping control.

Remark 6. By (3) and Assumption 2, it can be seen that $u(t-\tau)$ should be around the input $u$. If the time-delay $\tau$ is selected too large, the precision of approximation of simplified model will be reduced. So the selection of $\tau$ often requires experience. Theoretically, the smaller the $\tau$ the better precision of global approximation, the best precision of global approximation if $\tau=0$. But $u$ is control law to be solved, so it is unable to be realized. In order to obtain exact time-varying trim point, here, further improvement of above proposed method is given as follows. Considering lag property of the filtering as

$$
\dot{v}=-k_{\zeta} v+k_{\zeta} u
$$

then $\lim _{\lambda \rightarrow \infty} v=u$. This is a very good solution to the problem that $u(t-\tau)$ may not be around $u$. Here, $k_{\zeta} \rightarrow$ $\infty$ is only a rigorous expression for mathematics meanings, in general, $k_{\zeta} \in[5,50]$. The filter (7) is not unique. The filtering $v$ can be completely replaced by other filtering equation, such as higher-order differentiator [30] and integral filter [31].

\section{Sign Integral Terminal Sliding Mode Disturbance Observer}

In this section, the design process of the sign integral terminal sliding mode disturbance observer will be given. Firstly, the following auxiliary sign integral terminal sliding mode vector $s_{z}$ is introduced:

$$
s_{z}=e_{z}+\vartheta e_{z i}
$$

where $\vartheta>0$ is a design parameter, $e_{z}=x-z=\left[e_{z 1}, \ldots, e_{z n}\right]^{T}$ is auxiliary error, $z$ and $e_{z i}$ are given by

$$
\begin{gathered}
\dot{z}=f_{1}(x, u)+\widehat{D}, \\
\dot{e}_{z i}=\operatorname{sign}\left(e_{z}\right),
\end{gathered}
$$

where $e_{z i}$ is the integration of $\operatorname{sign}\left(e_{z}\right)$ and has the initial value $-e_{z}(0) / \vartheta$; and $\operatorname{sign}\left(e_{z i}\right)=\left[\operatorname{sign}\left(e_{z 1}\right), \ldots\right.$, $\left.\operatorname{sign}\left(e_{z n}\right)\right]^{T}$ for $e_{z i}(i=1, \ldots, n)$ being the $i$ th element of the auxiliary error vector $e_{z}$.

If $s_{z}(t)$ can keep at zero, such that $e_{z}(t)=-\vartheta e_{z i}(t)$, then dynamics (10) will be

$$
\dot{e}_{z i}(t)=\operatorname{sign}\left(e_{z}\right)(t) .
$$

Therefore, $e_{z i}(t)$ will converge to zero in the finite time $T_{f}$ :

$$
T_{f}=\frac{\left\|e_{z}(0)\right\|}{\vartheta} .
$$

Note that when the auxiliary sign integral terminal sliding vector $s_{z}$ satisfies $s_{z}(t)=0$, the convergence of the $e_{z}(t)$ is accomplished in the same time finite time (12) due to the fact that $e_{z}(t)=-\vartheta e_{z i}(t)$.

Next, to keep the system on the sign integral terminal sliding surface $s_{z}(t)=0$, the sliding mode disturbance estimate $\widehat{D}$ will be set to

$$
\widehat{D}=k_{z} s_{z}+\kappa \operatorname{sign}\left(s_{z}\right)+\vartheta \operatorname{sign}\left(e_{z}\right),
$$

where $k_{z}>0$ and $\kappa>\check{\epsilon}>0$ are design parameters.

Theorem 7. Considering the uncertain nonaffine nonlinear system (1) and supposing that Assumption 1 is available, the sign integral terminal sliding mode observer is designed according to (8)-(13). Then, auxiliary errors $e_{z}(t)$ and $e_{z i}(t)$ are guaranteed with finite-time convergence stability.

Proof. Based on (8)-(13), the sign integral terminal sliding mode dynamic equation (8) also can be expressed by

$$
\begin{aligned}
\dot{s}_{z} & =\dot{x}-\dot{z}+\vartheta \dot{e}_{z i} \\
& =f_{1}(x, u)+D-f_{1}(x, u)-\widehat{D}-\vartheta \operatorname{sign}\left(e_{z}\right)+\vartheta \dot{e}_{z i} \\
& =D-k_{z} s_{z}-\kappa \operatorname{sign}\left(s_{z}\right) .
\end{aligned}
$$

Choose the Lyapunov function candidate:

$$
V_{s}=\frac{1}{2} s_{z}^{T} s_{z}
$$

The time derivative of $V_{s}$ along the trajectories of the equation in (14) is

$$
\begin{aligned}
\dot{V}_{s} & =s_{z}^{T} \dot{s}_{z}=s_{z}^{T}\left(D-k_{z} s_{z}-\kappa \operatorname{sign}\left(s_{z}\right)\right) \\
& \leqslant-k_{z} s_{z}^{T} s_{z}+\left\|s_{z}\right\| \check{\epsilon}-\kappa s_{z}^{T} \operatorname{sign}\left(s_{z}\right)
\end{aligned}
$$

Under Assumption 1, considering design parameter $\kappa>\check{\epsilon}$, that is, $\kappa>\|D\|$, (16) can be modified as

$$
\dot{V}_{s} \leqslant-k_{z} s_{z}^{T} s_{z}=-2 k_{z} V_{s} .
$$

From (17), we can get the conclusion that if $s_{z} \neq 0$, then $\dot{V}_{s}<0$ is true. Thus, the auxiliary sliding vector $s_{z}$ of the sign integral terminal sliding mode disturbance observer is always kept on the surface $s_{z}(t)=0$. At the result, the auxiliary errors $e_{z}(t)$ and $e_{z i}(t)$ are guaranteed with finite-time convergence stability. This ends the proof. 
Remark 8. Comparing with the existing results [14], the proposed sign integral terminal sliding mode disturbance observer can guarantee the disturbance estimate error to converge to zero in the finite time. In addition, the advantages of proposed sign integral terminal sliding mode will be explained in Remarks 11 and 12.

Remark 9. It is worth noting that the known upper boundary of the dynamic error is required in the design of disturbance observer. However, upper boundary $\check{\epsilon}$ is difficult to be obtained in practice. So, the adaptive gain $\kappa$ in (13) is considered. There are many research results on adaptive gain [16]. For simple convenience, the detail is omitted.

\section{Fractional Integral Terminal Sliding Mode Control}

In this section, we develop the tracking control scheme for the case where all states are available using fractional integral terminal sliding mode control approach. Before the discussion, the tracking error is defined as $e(t)=y(t)-$ $y_{d}(t)$. Instead of using a linear sliding function, we introduce fractional integral terminal sliding mode below. First, the fractional integral terminal sliding mode function is defined as follows:

$$
\begin{gathered}
s(t)=e(t)+\breve{\alpha} e_{I}(t), \\
\dot{e}_{I}(t)=e^{q / p}(t),
\end{gathered}
$$

where $\breve{\alpha}>0 ; q$ and $p$ are positive odd integers with $p>q$; $e_{I}=\int_{0}^{t} e^{q / p}(\tau) d \tau$ is the integration of the $q / p$-fractional power of the tracking error $e$ with the initial value $-e(0) / \breve{\alpha}$; the nonlinear $e^{q / p}$ is obtained by the operation $e_{0}^{q / p}=$ $\left[e_{1}^{q / p} \operatorname{sign}\left(e_{1}\right), \ldots, e_{n}^{q / p} \operatorname{sign}\left(e_{n}\right)\right]^{T}$.

If the surface $s(t)=0$, based on definition (18), the fractional integral terminal sliding mode function can be expressed in the form

$$
s(t)=e(t)+\breve{\alpha} \int_{0}^{t} e^{q / p}(\tau) d \tau .
$$

At the same time, the integrator $e_{I}(t)$ can be modified as

$$
\dot{e}_{I}=-\breve{\alpha}^{q / p} e_{I}^{q / p}(t)
$$

From solving the error dynamic equation (20), the convergence time of $e_{I}$ is obtained as follows:

$$
T_{f}=\frac{\left\|e_{I}\right\|^{1-q / p}}{\breve{\alpha}^{q / p}(1-q / p)}=\frac{\|e(0)\|^{1-q / p}}{\breve{\alpha}(1-q / p)} .
$$

Meanwhile, the time spent for the convergence of the tracking error $e(t)$ is also $T_{f}$.

Next, to keep the system on the integral terminal sliding surface $s(t)=0$, we need to design control input vector $u$ for the system (2). Considering the time-varying simplified model (6), we modify the time derivative of $s(t)$ along the dynamics (6) as

$$
\dot{s}(t)=\dot{e}+\breve{\alpha} e_{I}=f+g u+O(\cdot)+D-\dot{y}_{d}+\breve{\alpha} \dot{e}_{I},
$$

where $f, g$, and $O(\cdot)$ are defined in Section 3.
According to Assumption 2, we will first consider the case when the control gain is nonsingular; that is, $|g| \neq 0$. Following that, our focus will be on the control design in the case when the control gain is singular; that is, $|g|=0$.

5.1. Case of Nonsingular Control Gain. In this subsection, we assume that $|g| \neq 0$ for the nonaffine nonlinear systems (2) with simplified model (6). Then, we consider the following the control vector $u$ as

$$
u=g^{-1}\left(-k s-f-\widehat{D}-\breve{\alpha} \dot{e}_{I}+\dot{y}_{d}-r_{s}\right),
$$

where $k>0$ is a design parameter; $\widehat{D}$ is defined in (13); to restrain the dynamic error $O(\cdot)$ from (6), the robust term $r_{s}$ is designed as

$$
r_{s}= \begin{cases}\frac{\varsigma s}{\|s\|}, & \|s\| \neq 0, \\ 0, & s=0,\end{cases}
$$

where $\varsigma>\|O(\cdot)\|$ is a design constant.

The above design procedure of the terminal sliding mode control can be summarized in the following theorem, which contains the results for disturbance-observer-based terminal sliding mode tracking control of uncertain nonaffine systems with external disturbance.

Theorem 10. Considering the uncertain nonaffine system (1) with the external disturbance and assuming that Assumptions 1 and 2 are available, nonaffine nonlinear approximation is given as (6) and the terminal sliding mode disturbance observer is designed as (8)-(13). If the proposed terminal sliding mode tracking control and the robust term are chosen as (23) and (24), then all signals of the closed-loop system are convergent in the finite time (21).

Proof. Choose the Lyapunov function candidate:

$$
V=\frac{1}{2} s^{T} s
$$

Under Assumption 2, substituting (23) and (24) into (22), the time derivative of $V$ along the trajectories of (22) is

$$
\begin{gathered}
\dot{V}=s^{T} \dot{s}=s^{T}\left(f+g u+D+O(\cdot)-\dot{y}_{d}+\breve{\alpha} \dot{e}_{I}\right) \\
=s^{T}\left(f-k s-f-\widehat{D}-\breve{\alpha} \dot{e}_{I}+\dot{y}_{d}\right. \\
\left.\quad-r_{s}+D+O(\cdot)-\dot{y}_{d}+\breve{\alpha} \dot{e}_{I}\right) \\
\leqslant-k s^{T} s+\|s\|\|O(\cdot)\|-\varsigma\|s\| \\
\leqslant-k s^{T} s=-2 k V .
\end{gathered}
$$

Since $s(0)=0$ and (26), the system is always kept on the fractional integral terminal sliding surface $s(0)=0$. As a result, the tracking error $e_{I}(t)$ and error $e(t)$ converge to zero in finite time (21). This concludes the proof. 
Remark 11. The characteristics of the proposed fractional integral terminal sliding mode control including (1) the finite convergence time can be easily adjusted according to (21); (2) the singular problem does not occur on the control law in contrast to traditional TSMC; (3) the system starts on the sliding mode surface $s=0$; that is, fast response is obtained.

Remark 12. Aside from the characteristics in Remark 11, the convergence time of the fractional integral terminal sliding mode control is calculable and analyzable in contrast to the high-order SMC. In comparison, the dynamic SMC only assures asymptotic stability.

Remark 13. In order to reduce chattering which is caused by discontinuous sign function, $\varsigma s /\|s\|$ in robust term (24) can be replaced by the continuous function $r_{s}$ defined by

$$
r_{s}=\frac{\varsigma s}{\|s\|+\epsilon}
$$

with $\epsilon=\epsilon_{0}+\epsilon_{1}\|e\|$, where $\epsilon_{0}$ and $\epsilon_{1}$ are two positive constants.

5.2. Case of Singular Control Gain. In Section 5.1, we assume that $|g(x)| \neq 0$ for the simplified model (6). However, there exists the feasibility of $|g(x)|=0$ at a moment in the practical system which leads to the control singularity. Thus, we propose the fractional integral terminal sliding mode control for the simplified model (6) with control singularity case in this subsection. Considering the control singularity, the control input vector $u$ is given by

$$
u=g(x, v)\left(g^{T}(x, v) g(x, v)+\lambda\right)^{-1} \check{u},
$$

where $\lambda>0$ is a design constant and $\check{u}$ will be given later.

It is clear that

$$
\begin{gathered}
g^{T}(x, v) g(x, v)\left(g^{T}(x, v) g(x, v)+\lambda\right)^{-1} \\
=1-\lambda\left(g^{T}(x, v) g(x, v)+\lambda\right)^{-1} .
\end{gathered}
$$

Substituting (28) and (29) into (6), we obtain

$$
\dot{x}=f(x, v)+\check{u}+D-\left(g^{T}(x, v) g(x, v)+\lambda\right)^{-1} \check{u}+O(\cdot) .
$$

According to (30), the compound disturbance can be modified as

$$
\bar{D}=D-\left(g^{T}(x, v) g(x, v)+\lambda\right)^{-1} \check{u}+O(\cdot) .
$$

Due to the unknown compound disturbance $\bar{D}$, the sign integral terminal sliding mode disturbance observer needs to be developed to estimate it. Thus, the similar auxiliary sliding mode is expressed:

$$
\begin{gathered}
s_{z}=e_{z}+a_{0} e_{z i}, \\
\dot{z}=f+\check{u}+\widehat{\bar{D}}, \\
\dot{e}_{z i}=\operatorname{sign}\left(e_{z i}\right),
\end{gathered}
$$

where $s_{z}, e_{z}, e_{z i}$, and $z$ are defined in Section 4 . Based on (32)-(34), the sign integral terminal sliding mode disturbance estimate $\widehat{\bar{D}}$ is given by

$$
\widehat{\bar{D}}=k_{z} s_{z}+\kappa \operatorname{sign}\left(s_{z}\right)+\vartheta \operatorname{sign}\left(e_{z}\right),
$$

where $\widehat{\bar{D}}$ is the estimate of compound disturbance $\bar{D}$.

Based on the sign integral terminal sliding mode disturbance observer, the fractional integral terminal sliding mode tracking control is designed as

$$
\check{u}=-k s-f-\widehat{\bar{D}}-\breve{\alpha} e^{q / p}+\dot{y}_{d} \text {, }
$$

where the parameters $k, \breve{\alpha}, q$, and $p$ are defined in (23) and (18), respectively.

The above design procedure and analysis can be summarized in the following theorem, which contains the results for the simplified model (6) with the control singularity case.

Theorem 14. Considering the uncertain nonaffine system (1) with the external disturbance and supposing that Assumptions 1 and 2 are available, nonaffine nonlinear approximation is given as (6) and the sign integral terminal sliding mode disturbance observer is designed as (32)-(35). If the proposed fractional integral terminal sliding mode control law is chosen as (36), then sliding mode surface will always keep at $s(t)=0$.

Proof. Considering the time-varying simplified model (6) with singular control gain, we modify the time derivative of $s(t)$ along the dynamics (30) as

$$
\dot{s}(t)=\dot{e}+\breve{\alpha} e_{I}=f+g u+O(\cdot)+D-\dot{y}_{d}+\breve{\alpha} \dot{e}_{I} .
$$

Choose the Lyapunov function candidate:

$$
V=\frac{1}{2} s^{T} s
$$

Substituting (36) into (37), the time derivative of $V$ along the trajectories of the equation in (37) is

$$
\begin{aligned}
\dot{V}= & s^{T} \dot{s}=s^{T}\left(f+\breve{u}+\bar{D}-\dot{y}_{d}+\breve{\alpha} \dot{e}_{I}\right) \\
= & s^{T}\left(f-k s-f-k_{z} s_{z}-\widehat{\bar{D}}\right. \\
& \left.\quad-\breve{\alpha} e^{q / p}+\dot{y}_{d}+\bar{D}-\dot{y}_{d}+\breve{\alpha} \dot{e}_{I}\right) \\
\leqslant & -k s^{T} s=-2 k V .
\end{aligned}
$$

According to $s(0)=0$ and (39), we can know that the system is always kept on the fractional integral terminal sliding surface $s(0)=0$. So, the tracking error $e_{I}(t)$ and error $e(t)$ converge to the equilibrium point in the finite time (21). This concludes the proof. 


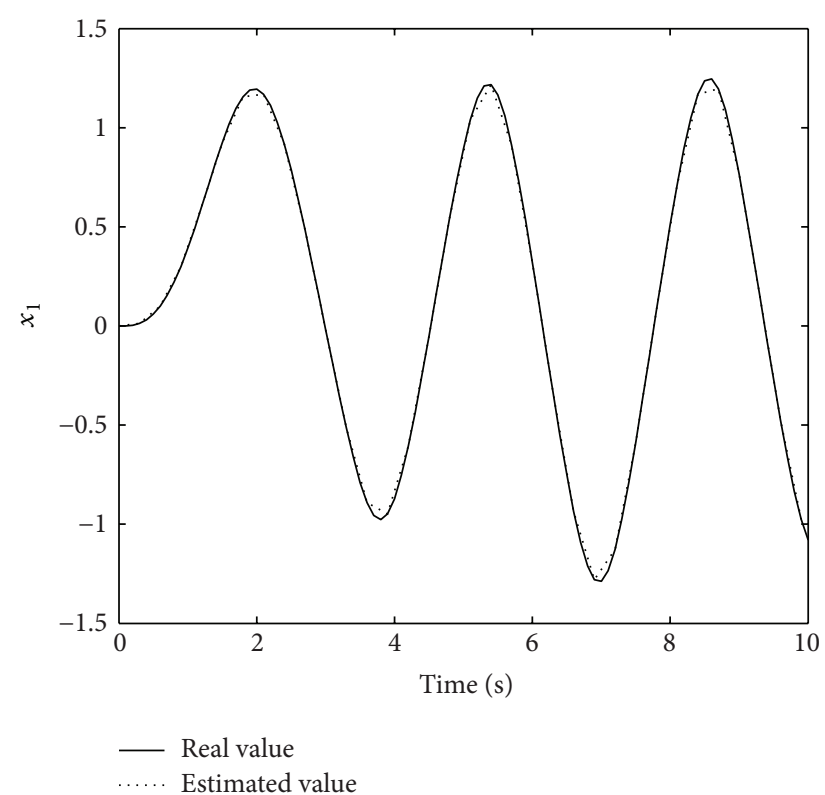

(a)

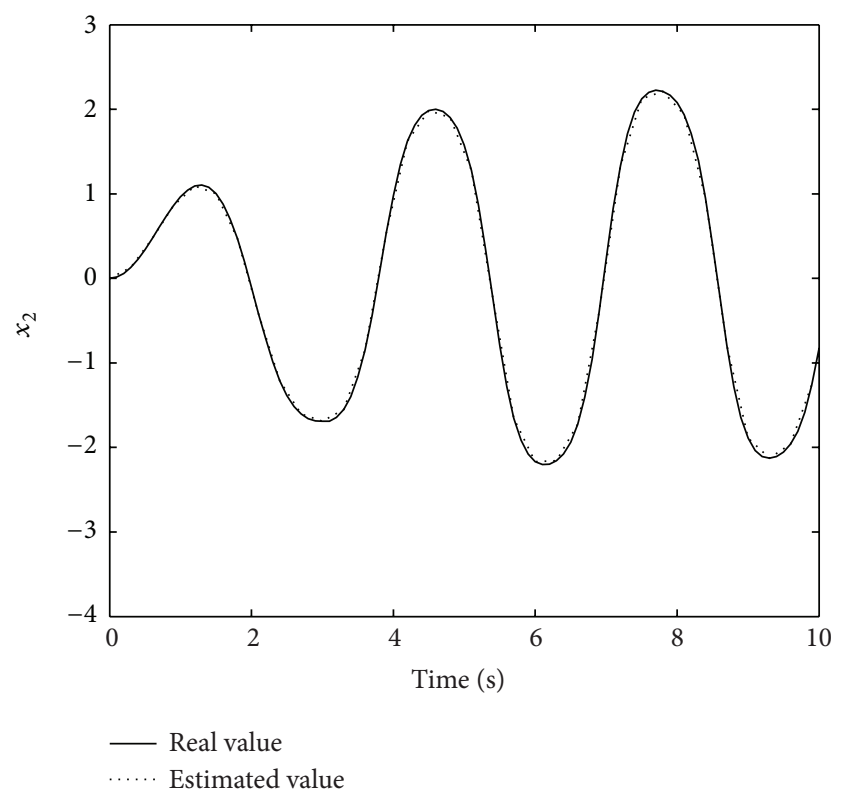

(b)

FIGURE 1: State response by the proposed approximation.

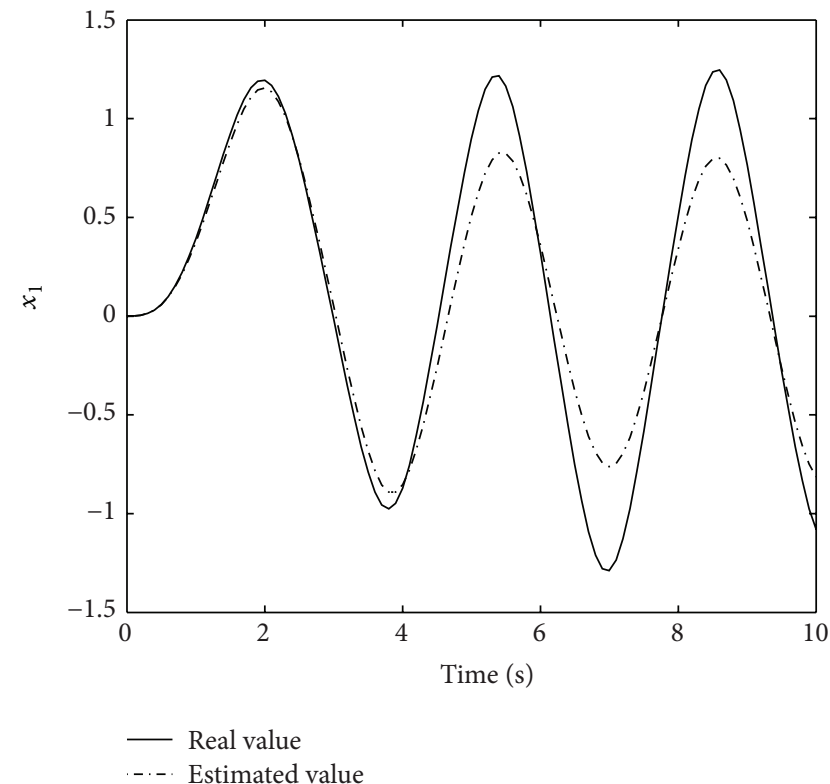

(a)

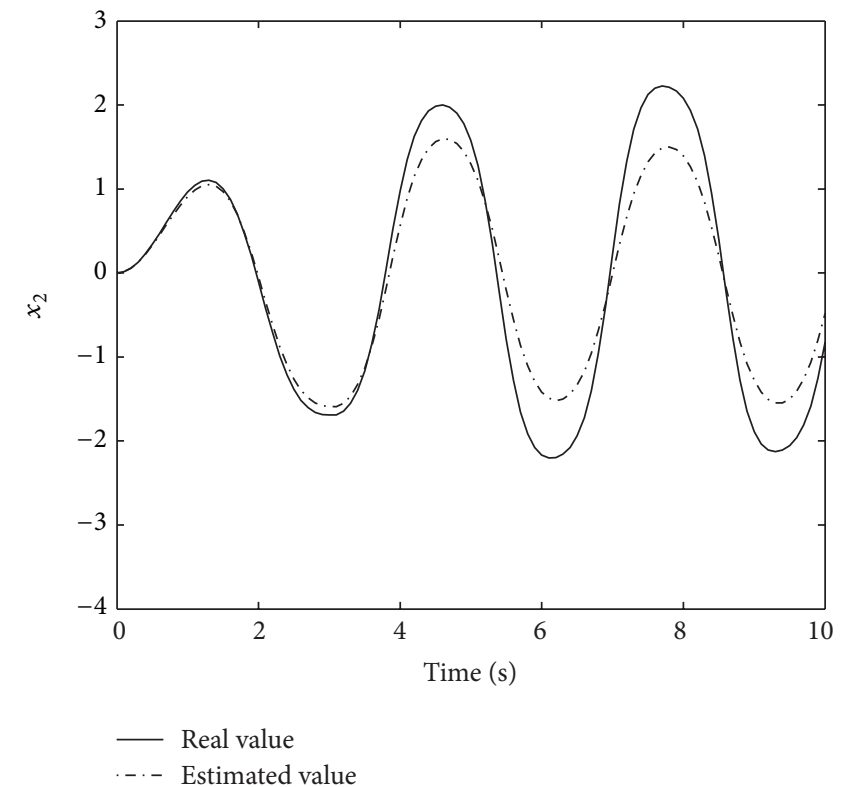

(b)

FIGURE 2: State response using the approach developed in [29].

Remark 15. From (31), the integrated effect of control singularity is treated as a part of the external disturbance which is approximated using the sign integral terminal sliding mode disturbance observer (33)-(35). Although the uncertain nonlinear system (1) has the feasibility of control singularity, Lyapunov analysis shows that the system is asymptotically convergent in the finite time under the proposed disturbanceobserver-based fractional integral terminal sliding mode control. In general, the design parameter $k_{z}$ should be chosen as a large positive constant to guarantee the design requirement of the proposed sliding mode disturbance observer. 


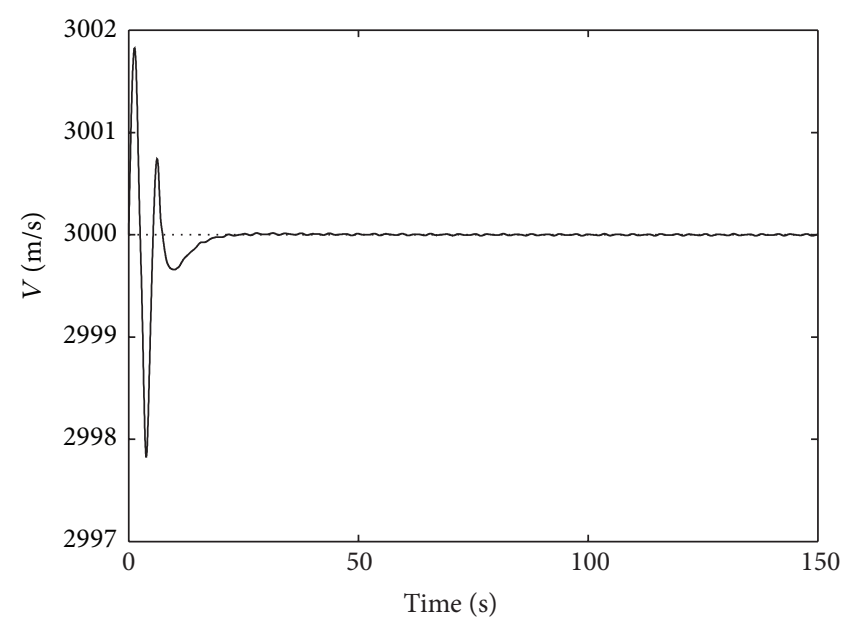

Reference

Tracking value

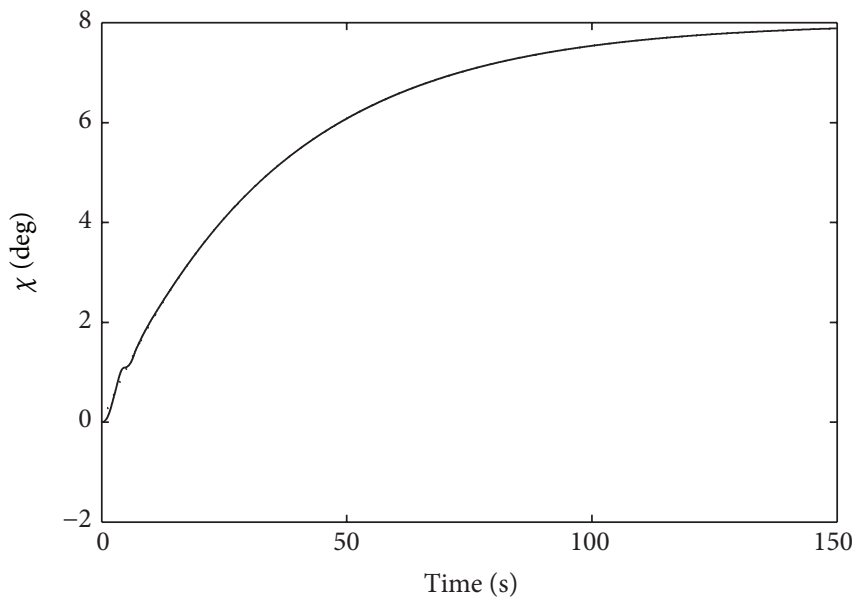

Reference

Tracking value

(a)

(b)

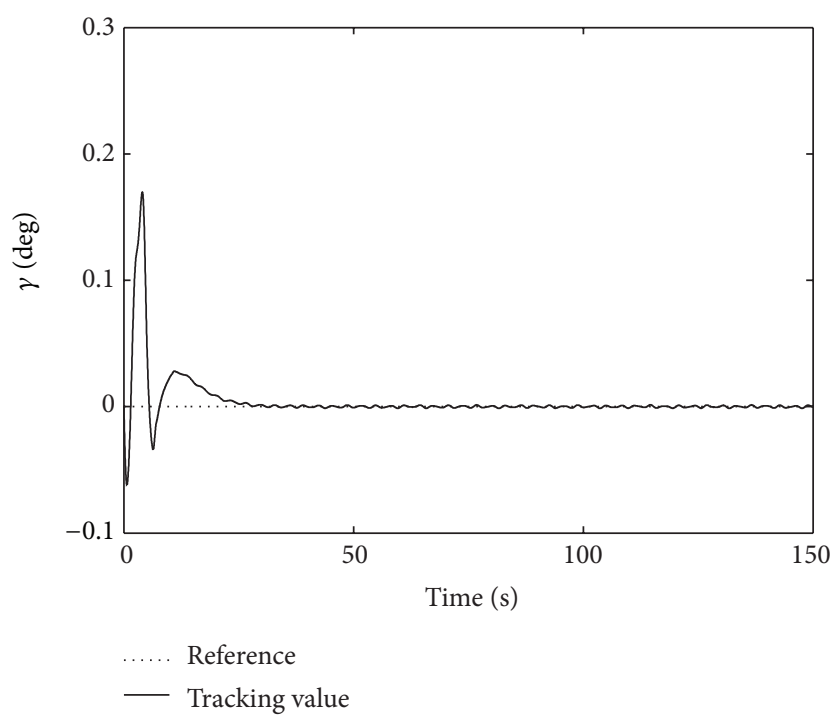

(c)

Figure 3: States $V, \chi$, and $\gamma$ follow desired command $V_{d}, \chi_{d}$, and $\gamma_{d}$ for near space vehicle system with coordinated turn.

\section{Simulation Results}

6.1. Simulation Example for Duffing-Holmes System. To verify the validity of the proposed nonaffine nonlinear approximation in Section 3, consider Duffing-Holmes system

$$
\begin{gathered}
\dot{x}_{1}=x_{2}, \\
\dot{x}_{2}=-p_{1} x_{1}-p_{2} x_{2}-x_{1}^{3}+h\left(x_{2}, u\right),
\end{gathered}
$$

where $p_{1}=0.2 \sin (10 t) ; p_{2}=0.2(1+\cos (5 t)) ; h\left(x_{2}, u\right)=u^{3}+$ $\left(2+\cos \left(x_{2}\right)\right) u$ is a nonaffine control term.
The initial conditions are chosen as $\left[x_{1}(0), x_{2}(0)\right]^{T}=0$, and the simulation time is chosen as $t=30 \mathrm{~s}$. we define control input value $u$ as

$$
u(t)= \begin{cases}0.1 \sin (2 t), & t \leqslant 7, \\ \sin (2 t), & 7<t \leqslant 30 .\end{cases}
$$

As compared with the existing approximate method, we adopt the method of [29] at the local working point $u=0$ for nonaffine nonlinear systems (2). Then, we get the affine nonlinear approximation as follows:

$$
\begin{gathered}
\dot{x}_{1}=x_{2}, \\
\dot{x}_{2}=-p_{1} x_{1}-p_{2} x_{2}-x_{1}^{3}+\left(2+\cos \left(x_{2}\right)\right) u .
\end{gathered}
$$


By using the proposed approximation method in Section 3, the affine nonlinear approximation is

$$
\begin{gathered}
\dot{x}_{1}=x_{2}, \\
\dot{x}_{2}=-p_{1} x_{1}-p_{2} x_{2}-x_{1}^{3}-2 \zeta^{3}+3 \zeta^{2} u+\left(2+\cos \left(x_{2}\right)\right) u, \\
\dot{\zeta}=-k_{\zeta} \zeta+k_{\zeta} u, \quad \zeta(0)=0 .
\end{gathered}
$$

The designed constant in (43) is chosen as $k_{\zeta}=100$. Simulation results are shown in Figures 1 and 2, respectively.

From Figures 1 and 2, it is observed that two methods have the same approximation during working point $u=0$. On the other hand, when the working point stay away from $u=0$, changes. Therefore, from the global approximation, the proposed method is better than the method of [29].

6.2. Simulation Example for 6DOF Near Space Vehicle Dynamics with Coordinated Turn. To verify the validity of the fractional integral terminal sliding mode control, the differential equations governing the near space vehicle (NSV) dynamics with coordinated turn are given by

$$
\begin{gathered}
\dot{x}=V \cos \gamma \cos \chi \\
\dot{y}=V \cos \gamma \sin \chi \\
\dot{z}=V \sin \gamma \\
p t \dot{V}=f_{v}=\frac{1}{M}(T \cos \alpha-D-M g \sin \gamma) \\
\dot{\chi}=f_{\chi}=\frac{1}{M V \cos \gamma}(L \sin \mu+Y \cos \mu+T \sin \mu \sin \alpha) \\
\dot{\gamma}=f_{\gamma}=\frac{1}{M V}(L \cos \mu-Y \sin \mu \\
-M g \cos \gamma+T \cos \mu \sin \alpha)
\end{gathered}
$$

where the three position variables $(x, y, z)$ in the inertial frame, airspeed $(V)$, fight path angle $(\gamma)$, and flight path heading $(\chi)$ are the six state variables; thrust $(T)$, attack angle $(\alpha)$, and roll angle $(\mu)$ are the control variables; the drag force $(D)$, lift force $(L)$, and lateral force $(Y)$ are expressed as follows:

$$
D=\widehat{q} S C_{D}, \quad L=\widehat{q} S C_{L}, \quad Y=\widehat{q} S C_{Y},
$$

where $\widehat{q}=0.5 \rho V^{2}$ is dynamic pressure, $S$ is reference area, $C_{D}=C_{D \alpha}+C_{D \delta_{e}} \delta_{e}+C_{D \delta_{a}} \delta_{a}+C_{D \delta_{r}} \delta_{r}+C_{D \delta_{c}} \delta_{c}, C_{L}=C_{L \alpha}+$ $C_{L \delta_{e}} \delta_{e}+C_{L \delta_{a}} \delta_{a}+C_{L \delta_{c}} \delta_{c}$, and $C_{Y}=C_{Y \beta} \beta+C_{Y \delta_{e}} \delta_{e}+C_{Y \delta_{\alpha}} \delta_{\alpha}+$ $C_{Y \delta_{r}} \delta_{r}$. In this paper, we will assume that the parameters $C_{D \alpha}$, $C_{D \delta_{e}}, C_{D \delta_{a}}, C_{D \delta_{r}}, C_{D \delta_{c}} \delta_{c}, C_{L \alpha}, C_{L \delta_{e}}, C_{L \delta_{a}}, C_{L \delta_{c}}, C_{Y \beta}, C_{Y \delta_{e}}$, $C_{Y \delta_{\alpha}}$, and $C_{Y \delta_{r}}$ are uncertain, while the description of the variables are shown in [32].

In this paper, we will focus on the model of rate dynamics, that is, (47)-(49). So, to put the above equations in the form of (1), we define $x=\left[x_{1}, x_{2}, x_{3}\right]^{T}=[V, \chi, \gamma]^{T}, u=$ $\left[u_{1}, u_{2}, u_{3}\right]^{T}=[T, \alpha, \mu]^{T}$. Hence the rate equations become

$$
\dot{x}=f(x, u),
$$

where $f=\left[f_{v}, f_{\chi}, f_{\gamma}\right]^{T}$ is defined in (47)-(49). By using the proposed approximated model in Section 3, we have

$$
\begin{gathered}
\dot{x}=\bar{f}+g u_{\zeta}+O(\cdot), \\
u_{\zeta}=-k_{\zeta} u_{\zeta}+k_{\zeta} u, \quad u_{\zeta}(0)=0,
\end{gathered}
$$

where $\bar{f}=\breve{f}\left(x, u_{\zeta}\right)-g_{p} u_{\zeta}, u_{\zeta}=\left[u_{\zeta 1}, u_{\zeta 2}, u_{\zeta 3}\right]^{T}, k_{\zeta}$ is $s$ design parameter, $\breve{f}\left(x, u_{\zeta}\right)=\left[\breve{f}_{v}, \breve{f}_{\chi}, \breve{f}_{\gamma}\right], \breve{f}_{v}, \breve{f}_{\chi}, \breve{f}_{\gamma}$, and $g_{p}$ are expressed as

$$
\begin{aligned}
& \breve{f}_{v}=\frac{1}{M}\left(u_{\zeta 1} \cos x_{2}-D-M g \sin x_{3}\right), \\
& \breve{f}_{\chi}=\frac{1}{M x_{1} \cos x_{3}}\left(L \sin u_{\zeta 3}+Y \cos u_{\zeta 3}+T \sin u_{\zeta 3} \sin u_{\zeta 2}\right) \text {, } \\
& \breve{f}_{\gamma}=\frac{1}{M x_{1}}\left(L \cos u_{\zeta 3}-Y \sin u_{\zeta 3}-M g \cos x_{3}+u_{\zeta 1} \cos u_{\zeta 3} \sin u_{\zeta 2}\right) \text {, } \\
& g_{p}=\left[\begin{array}{ccc}
\frac{\cos u_{\zeta 2}}{M} & -\frac{u_{\zeta 1} \sin u_{\zeta 2}}{M} & 0 \\
\frac{1}{M u_{\zeta 1} \cos x_{3}} \sin u_{\zeta 2} \sin u_{\zeta 3} & \frac{1}{M u_{\zeta 1} \cos x_{3}} u_{\zeta 1} \cos u_{\zeta 2} \sin u_{\zeta 3} & \frac{1}{M u_{\zeta 1} \cos x_{3}} \sin u_{\zeta 2} \cos u_{\zeta 3} \\
\frac{1}{M u_{\zeta 1}} \sin u_{\zeta 2} \cos u_{\zeta 3} & \frac{1}{M u_{\zeta 1}} u_{\zeta 1} \cos u_{\zeta 2} \cos u_{\zeta 3} & -\frac{1}{M u_{\zeta 1}} u_{\zeta 1} \sin u_{\zeta 2} \sin u_{\zeta 3}
\end{array}\right] .
\end{aligned}
$$




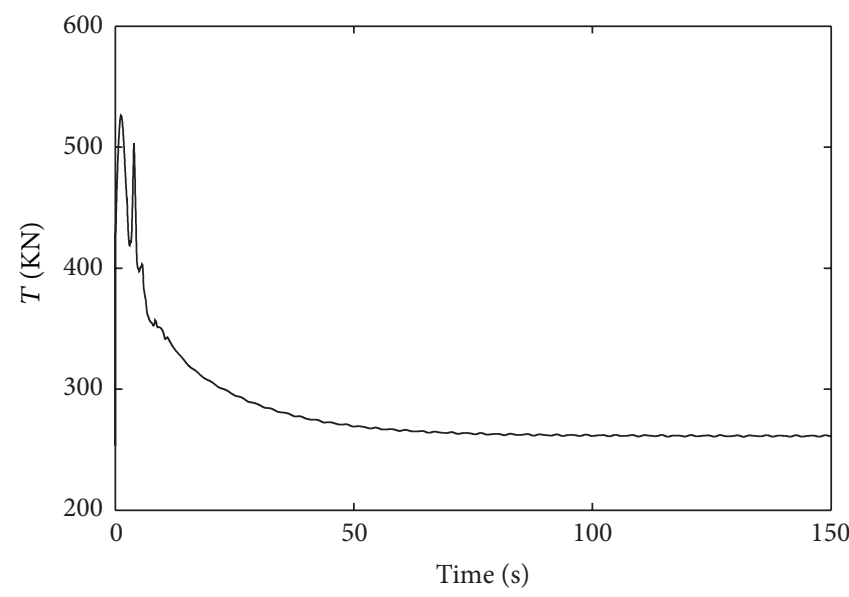

(a)

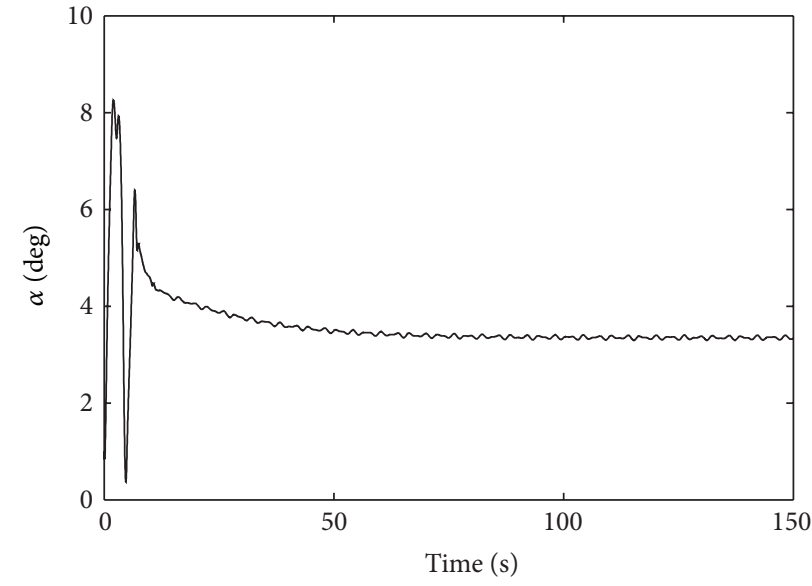

(b)

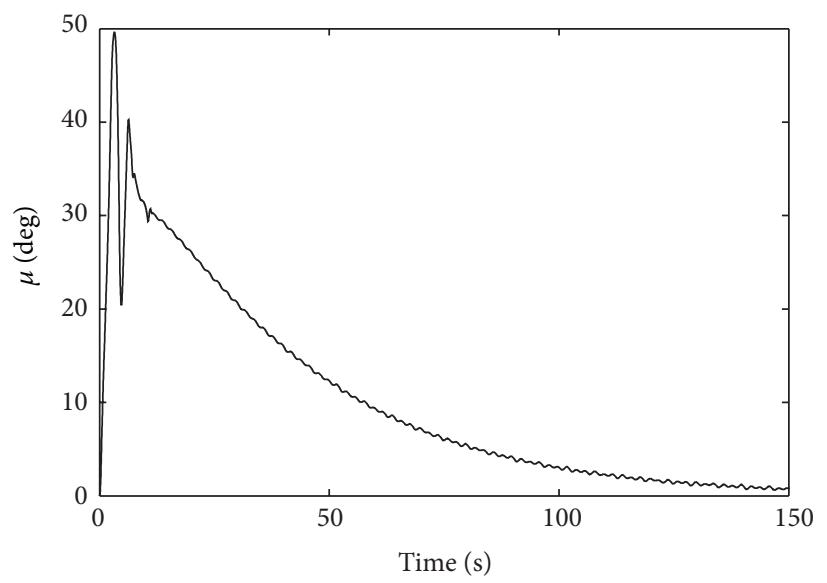

(c)

FIGURE 4: Control input for near space vehicle system with coordinated turn.

The initial state conditions are arbitrarily chosen as $M=$ $136080 \mathrm{Kg}, V(0)=3000 \mathrm{~m} / \mathrm{s}, \chi(0)=\gamma(0)=0 \mathrm{deg}, T(0)=$ $240 \mathrm{KN}, \alpha(0)=1 \mathrm{deg}$, and $\mu(0)=0 \mathrm{deg}$.

The desired command is considered as $V_{d}=3000 \mathrm{~m} / \mathrm{s}$, $\chi_{d}=8 \mathrm{deg}$, and $\gamma_{d}=0 \mathrm{deg}$. In order to ensure the smoothness of airspeed change, we choose the filter as (7), in which the parameter is chosen as $k_{\zeta}=0.03$.

To estimate the uncertainty, we apply integral terminal sliding mode disturbance observer in Section 4 and set the parameters as $k_{z}=5, \vartheta=2$, and $\kappa=0.5$. Furthermore, to demonstrate the effectiveness of the proposed integral terminal sliding mode control, the design parameters are chosen as $\breve{\alpha}=1.5, p=5, q=3, k=2$, and $\varsigma=0.3$. The tracking results are shown in Figures 3 and 4 . Although, there exists uncertain in the system, the tracking performance is still satisfactory and tracking error converges to zero quickly.

From these simulation results of two cases, we can obtain that the proposed method is valid. And the developed sign integral terminal sliding mode disturbance observer can modify the control performance of the fractional integral terminal sliding mode control.

\section{Conclusions}

In this paper, the disturbance-observer-based terminal sliding mode tracking control has been proposed for a class of uncertain nonaffine nonlinear systems. To design tracking controller, an on-line approximation has been proposed for a class of nonaffine nonlinear systems. To improve the ability of the disturbance attenuation and system performance robustness, the sign integral terminal sliding mode disturbance observer has been developed to approximate the system disturbance in the finite time. Based on the output of the disturbance observer, the disturbance-observer-based fractional integral terminal sliding mode tracking control has been presented for the uncertain nonlinear system with the timevarying external disturbance. By innovating the fractional error integration, finite-time convergence of tracking errors and integral errors is achieved without singular problem. Furthermore, the finite convergence time is easily calculated in contrast to the traditional high-order sliding mode control. The stability of the closed-loop system has been proved using rigorous Lyapunov analysis. Finally, simulation results have been used to illustrate the effectiveness of the proposed robust 
terminal sliding mode tracking control scheme. In addition, based on the proposed approach, how to relax Assumption 1 is our future works. At the same time, fault-tolerant control for a class of nonaffine nonlinear systems is also our future works.

\section{Acknowledgments}

This work was supported by Natural Science Foundation of Shandong Province under Grant ZR2012F Q030. The authors wish to thank the reviewers for their constructive comments and suggestions which have helped to improve the presentation of the paper.

\section{References}

[1] W.-Y. Wang, Y.-H. Chien, Y.-G. Leu, and T.-T. Lee, "Adaptive T-S fuzzy-neural modeling and control for general MIMO unknown nonaffine nonlinear systems using projection update laws," Automatica, vol. 46, no. 5, pp. 852-863, 2010.

[2] X. Su, P. Shi, and L. Wu, "A novel control design on discrete-time Takagi-Sugeno fuzzy systems with time-varying delays," IEEE Transactions on Fuzzy Systems, vol. 20, no. 6, pp. 655-671, 2013.

[3] D. Xu, B. Jiang, and P. Shi, "Nonlinear actuator fault estimation observer: an inverse system approach via a T-S fuzzy model," International Journal of Applied Mathematics and Computer Science, vol. 22, no. 1, pp. 183-196, 2012.

[4] S. Bououden, M. Chadli, and F. Allouani, "A new approach for fuzzy predictive adaptive controller design using particle swarm optimization algorithm," International Journal of Innovative Com-Puting, Information and Control, vol. 9, no. 9, pp. 37413758, 2013.

[5] J. Wen and C.-S. Jiang, "Adaptive fuzzy control for a class of chaotic systems with nonaffine inputs," Communications in Nonlinear Science and Numerical Simulation, vol. 16, no. 1, pp. 475-492, 2011.

[6] L. Wu, W. X. Zheng, and H. J. Gao, "Dissipativity-based sliding mode control of switched stochastic systems," IEEE Transactions on Automatic Control, vol. 58, no. 3, pp. 785-793, 2013.

[7] H. Q. Wang, B. Chen, and C. Lin, "Adaptive neural tracking control for a class of stochastic nonlinear systems with unknown dead-zone," International Journal of Innovative Computing, Information and Control, vol. 9, no. 8, pp. 3257-3269, 2013.

[8] G. Bartolini, E. Punta, and T. Zolezzi, "Simplex methods for nonlinear uncertain sliding-mode control," IEEE Transactions on Automatic Control, vol. 49, no. 6, pp. 922-933, 2004.

[9] E. N. Johnson, A. J. Calise, M. D. Curry, K. D. Mease, and J. Eric Corban, "Adaptive guidance and control for autonomous hypersonic vehicles," Journal of Guidance, Control, and Dynamics, vol. 29, no. 3, pp. 725-737, 2006.

[10] L. Wu, X. Su, and P. Shi, "Sliding mode control with bounded $L_{2}$ gain performance of Markovian jump singular time-delay systems," Automatica, vol. 48, no. 8, pp. 1929-1933, 2012.

[11] C. Mou, C.-S. Jiang, J. Bin, and Q.-X. Wu, "Sliding mode synchronization controller design with neural network for uncertain chaotic systems," Chaos, Solitons and Fractals, vol. 39, no. 4, pp. 1856-1863, 2009.

[12] V. I. Utkin and A. S. Poznyak, "Adaptive sliding mode control with application to super-twist algorithm: equivalent control method," Automatica, vol. 49, no. 1, pp. 39-47, 2013.
[13] A. Polyakov and A. Poznyak, "Reaching time estimation for "super-twisting" second order sliding mode controller via lyapunov function designing," IEEE Transactions on Automatic Control, vol. 54, no. 8, pp. 1951-1955, 2009.

[14] Y.-S. Lu, "Sliding-mode disturbance observer with switchinggain adaptation and its application to optical disk drives," IEEE Transactions on Industrial Electronics, vol. 56, no. 9, pp. 37433750, 2009.

[15] H. Lee and V. I. Utkin, "Chattering suppression methods in sliding mode control systems," Annual Reviews in Control, vol. 31, no. 2, pp. 179-188, 2007.

[16] F. Plestan, Y. Shtessel, V. Bregeault, and A. Poznyak, "New methodologies for adaptive sliding mode control," International Journal of Control, vol. 83, no. 9, pp. 1907-1919, 2010.

[17] M. Chen, Q. X. Wu, and R. X. Cui, "Terminal sliding mode tracking control for a class of SISO uncertain nonlinear systems," ISA Transactions, vol. 52, no. 2, pp. 198-206, 2013.

[18] L. Qi and H. B. Shi, "Adaptive position tracking control of permanent magnet synchronous motor based on RBF fast terminal sliding mode control," Neurocomputing, vol. 115, no. 4, pp. 23-30, 2013.

[19] V. Nekoukar and A. Erfanian, "Adaptive fuzzy terminal sliding mode control for a class of MIMO uncertain nonlinear systems," Fuzzy Sets and Systems, vol. 179, no. 1, pp. 34-49, 2011.

[20] H. Komurcugil, "Adaptive terminal sliding-mode control strategy for DC-DC buck converters," ISA Transactions, vol. 51, no. 6, pp. 673-681, 2012.

[21] C.-C. Yang, "Synchronization of second-order chaotic systems via adaptive terminal sliding mode control with input nonlinearity," Journal of the Franklin Institute, vol. 349, no. 6, pp. 20192032, 2012.

[22] Y. Feng, X. Yu, and Z. Man, "Non-singular terminal sliding mode control of rigid manipulators," Automatica, vol. 38, no. 12, pp. 2159-2167, 2002.

[23] D. Zhao, S. Li, and F. Gao, "A new terminal sliding mode control for robotic manipulators," in Proceedings of the 17th IFAC World Congress, pp. 9888-9893, Seoul, Korea, July 2008,

[24] S. S. Ge and K. P. Tee, "Approximation-based control of nonlinear MIMO time-delay systems," Automatica, vol. 43, no. 1, pp. 31-43, 2007.

[25] S. S. Ge, G. Y. Li, J. Zhang, and T. H. Lee, "Direct adaptive control for a class of MIMO nonlinear systems using neural networks," IEEE Transactions on Automatic Control, vol. 49, no. 11, pp. 2001-2006, 2004.

[26] M. Chen, S. S. Ge, and B. V. E. How, "Robust adaptive neural network control for a class of uncertain MIMO nonlinear systems with input nonlinearities," IEEE Transactions on Neural Networks, vol. 21, no. 5, pp. 796-812, 2010.

[27] H. H. Chiang, Y. L. Chen, and T. T. Lee, "Multi-stage with neuro-fuzzy approach for efficient on-road speed sign detection and recognition," International Journal of Innovative Computing, Information and Control, vol. 9, no. 7, pp. 2919-2939, 2013.

[28] Z. Tong and F. Feng-li, "Neural network-based adaptive output feedback control for MIMO non-affine systems," Neural Computing and Applications, vol. 21, no. 1, pp. 145-151, 2012.

[29] J.-H. Park, G.-T. Park, S.-H. Kim, and C.-J. Moon, "Direct adaptive self-structuring fuzzy controller for nonaffine nonlinear system," Fuzzy Sets and Systems, vol. 153, no. 3, pp. 429-445, 2005.

[30] X. Wang, Z. Chen, and G. Yang, "Finite-time-convergent differentiator based on singular perturbation technique," IEEE 
Transactions on Automatic Control, vol. 52, no. 9, pp. 1731-1737, 2007.

[31] C.-Y. Li, W.-X. Jing, and C.-S. Gao, "Adaptive backsteppingbased flight control system using integral filters," Aerospace Science and Technology, vol. 13, no. 2-3, pp. 105-113, 2009.

[32] Y. L. Du, Study of Nonlinear Adaptive Attitude and Trajectory Control for Near Space Vehicles, Nanjing University of Aeronautics and Astronautics, NanJing, China, 2010. 


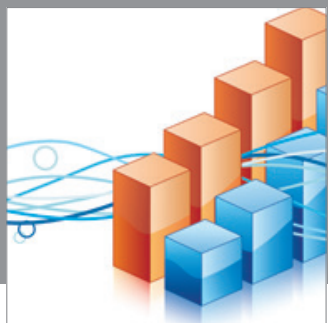

Advances in

Operations Research

mansans

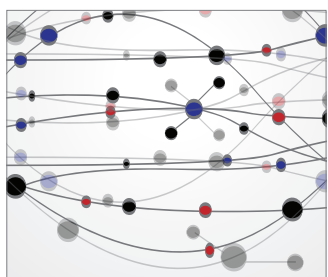

The Scientific World Journal
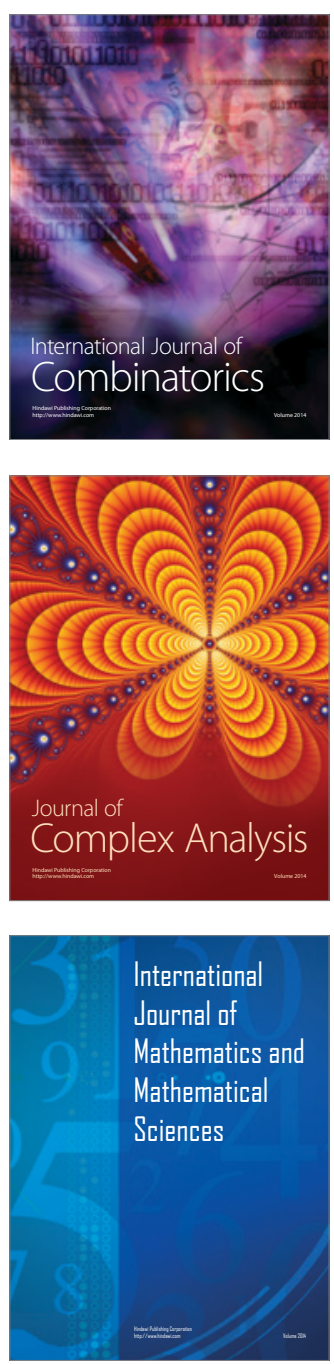
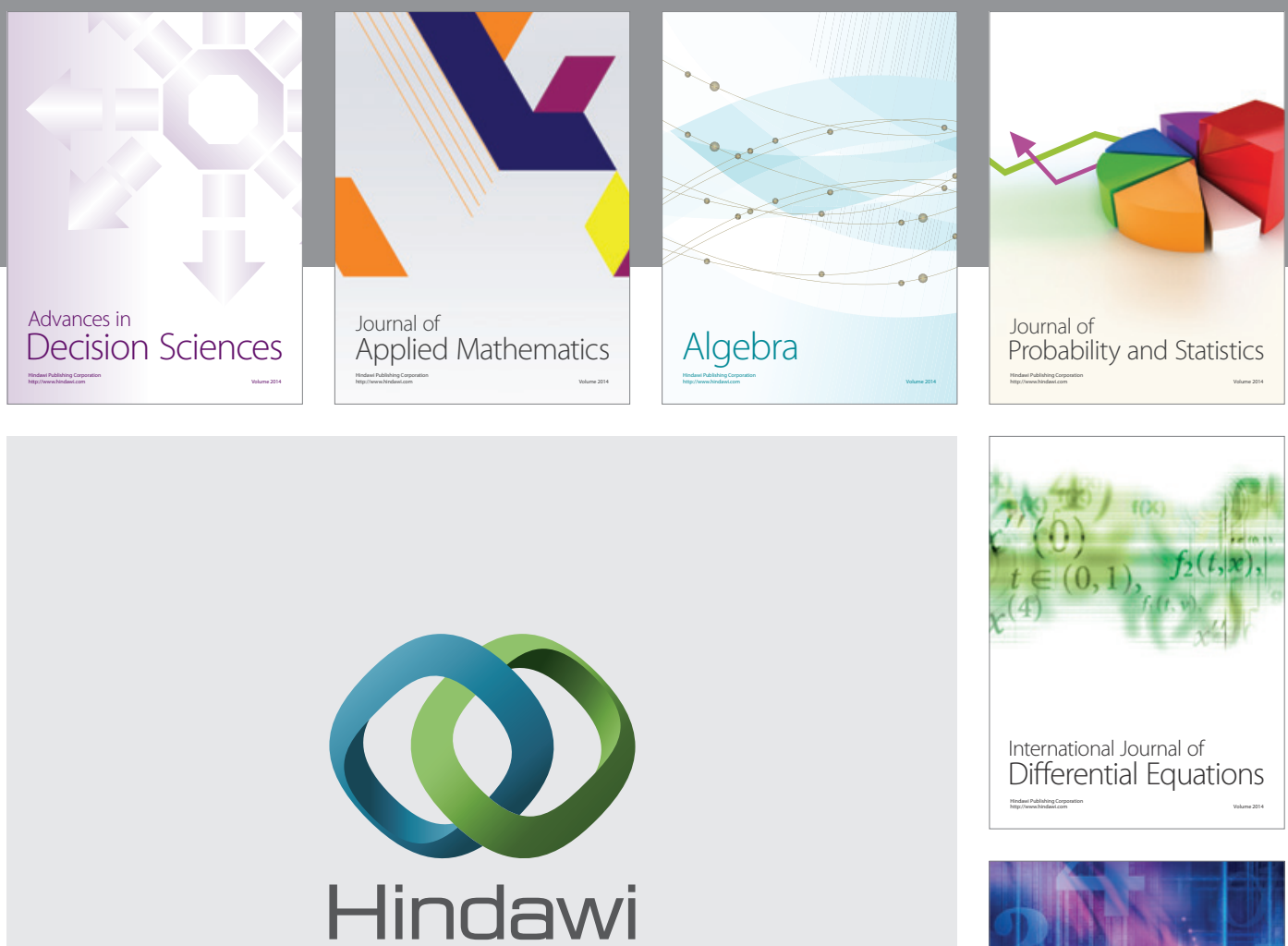

Submit your manuscripts at http://www.hindawi.com
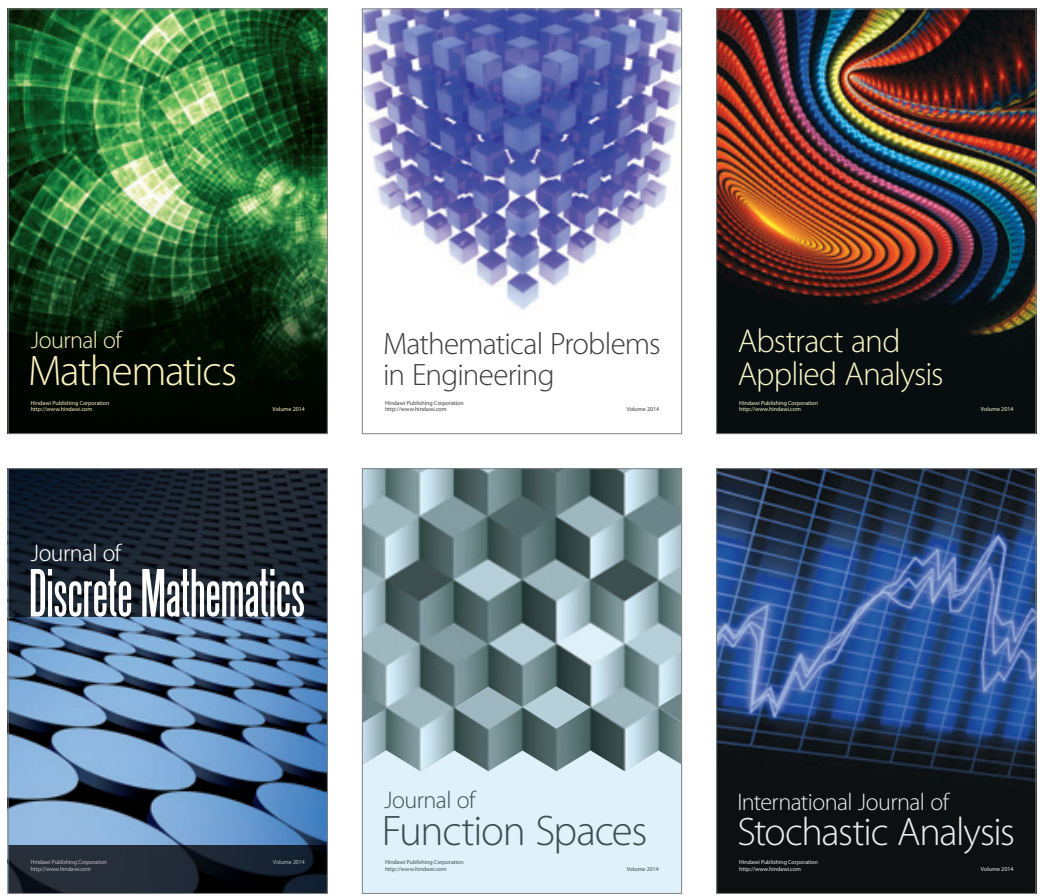

Journal of

Function Spaces

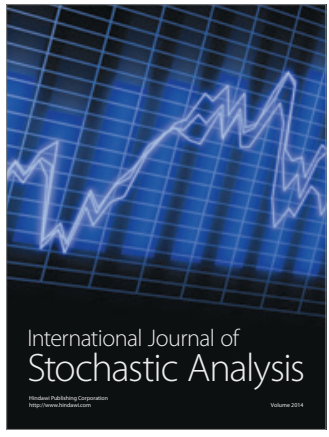

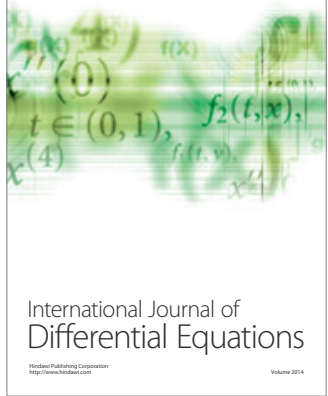
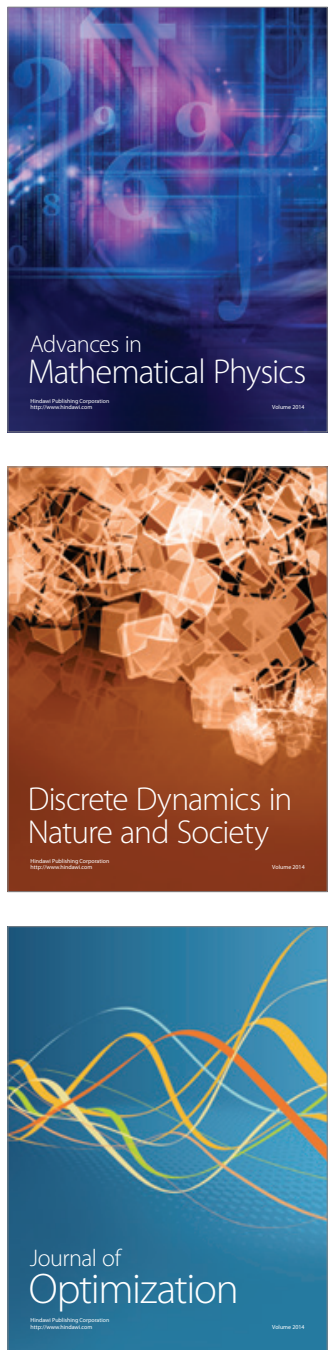